\title{
Photonic molecules with a tunable inter-cavity gap
}

\author{
Tobias Siegle $^{1}$, Stefan Schierle ${ }^{1}$, Sarah Kraemmer ${ }^{1}$, Benjamin Richter ${ }^{2}$, Sentayehu F Wondimu ${ }^{3,4}$, Peter Schuch ${ }^{3}$, \\ Christian Koos ${ }^{3,4}$ and Heinz Kalt ${ }^{1}$
}

Optical micro-resonators have broad applications. They are used, for example, to enhance light-matter interactions in optical sensors or as model systems for investigating fundamental physical mechanisms in cavity quantum electrodynamics. Coupling two or more micro-cavities is particularly interesting as it enlarges the design freedom and the field of application. In this context, achieving tunability of the coupling strength and hence the inter-cavity gap is of utmost importance for adjusting the properties of the coupled micro-resonator system. In this paper, we report on a novel coupling approach that allows highly precise tuning of the coupling gap of polymeric micro-resonators that are fabricated side by side on a common substrate. We structure goblet-shaped whispering-gallery-mode resonators on an elastic silicone-based polymer substrate by direct laser writing. The silicone substrate is mechanically stretched in order to exploit the lateral shrinkage to reduce the coupling gap. Incorporating a laser dye into the micro-resonators transforms the cavities into micro-lasers that can be pumped optically. We have investigated the lasing emission by micro-photoluminescence spectroscopy, focusing on the spatial localization of the modes. Our results demonstrate the formation of photonic molecules consisting of two or even three resonators, for which the coupling strengths and hence the lasing performance can be precisely tuned. Flexibility and tunability are key elements in future photonics, making our approach interesting for various photonic applications. For instance, as our coupling approach can also be extended to larger cavity arrays, it might serve as a platform for tunable coupled-resonator optical waveguide devices. Light: Science \& Applications (2017) 6, e16224; doi:10.1038/Isa.2016.224; published online 24 March 2017

Keywords: coupled resonators; photonic molecules; micro-lasers; tunable coupling gap; whispering gallery mode resonators

\section{INTRODUCTION}

Whispering-gallery-mode (WGM) resonators have emerged as versatile photonic structures for fundamental investigations in quantum optics $^{1-3}$, as well as for applications in optical sensing ${ }^{4-9}$, laser emission ${ }^{10-12}$ and telecommunication technology ${ }^{13-15}$. Among the various types of micro-cavities ${ }^{16,17}$, WGM resonators are most ideally suited for optical coupling to form so-called photonic molecules (PMs). In PMs, optical modes interact to create super-modes extending over the entire ensemble of coupled cavities ${ }^{18,19}$. This capability further enhances the performance of WGM resonators and broadens the scope of their applications. In lasing devices, coupling to PMs enables mode selection and single-mode emission $^{20-22}$ owing to the Vernier effect, which also enhances the sensitivity of cascaded WGM micro-ring resonators ${ }^{23,24}$. Coupled dye-doped polymer micro-fibers can be used as enhanced refractive index sensors ${ }^{22}$. Furthermore, the angular emission profile of PMs can be tailored-even to be uni-directional ${ }^{25,26}$. Coupled resonators also show an enhanced $Q$ factor ${ }^{27,28}$. They are used as optical filters and switches, where line-shape and bandwidth tuning lead to improved optical filtering responses ${ }^{29-31}$. In addition, the increased electromagnetic field in the coupling gap associated with the supermodes results in an enhanced sensitivity for PM sensors ${ }^{20,32}$.

The up-scaling of photonic molecules to arrays of coupled resonators leads to so-called coupled-resonator optical waveguides (CROWs). CROWs have unique dispersion properties allowing their usage as compact delay lines ${ }^{33-35}$. The group velocity of light propagating in a CROW is dependent on the coupling coefficient $\kappa$, which describes the coupling strength between the individual microcavities ${ }^{36,37}$. Realizing adjustable gap widths and coupling coefficients would hence pave the way to tunable and switchable CROW devices.

Thus far, flexible coupling between waveguides and optical microresonators has been established, for example, by the use of microelectrical mechanical systems ${ }^{38,39}$. Tunable photonic crystals have also been achieved by micro-electrical mechanical system approaches ${ }^{40,41}$. However, direct dynamical coupling among adjacent resonators has not been achieved in a viable way. The micro-cavities are typically structured on rigid substrates by lithography and are therefore intrinsically inflexible after fabrication ${ }^{20,25,42}$, excluding dynamic tunability in general. Sometimes, additional surface modification after fabrication is required to reduce the gap width to the order of the

${ }^{1}$ Institute of Applied Physics, Karlsruhe Institute of Technology (KIT), 76128 Karlsruhe, Germany; ${ }^{2}$ Zoological Institute, Karlsruhe Institute of Technology (KIT), 76128 Karlsruhe, Germany; ${ }^{3}$ Institute of Microstructure Technology, Karlsruhe Institute of Technology (KIT), 76128 Karlsruhe, Germany and ${ }^{4}$ Institute of Photonics and Quantum Electronics, Karlsruhe Institute of Technology (KIT), 76128 Karlsruhe, Germany

Correspondence: T Siegle, Email: tobias.siegle@kit.edu

Received 5 April 2016; revised 31 August 2016; accepted 5 September 2016; accepted article preview online 7 September 2016 
wavelength, which is essential for optical coupling ${ }^{20}$. The tuning of coupling gaps can be achieved when individual cavities are fixed to nano-positioning systems ${ }^{43,44}$. However, this approach is experimentally challenging, as precise alignment is essential and vulnerable to mechanical vibrations. It is further limited to PMs consisting of only two micro-resonators and is not scalable to CROWs.

In this article, we present a novel coupling approach for the flexible formation of PMs from WGM micro-resonators. This approach is based on resonator fabrication by direct laser writing (DLW) on elastomer substrates. It enables the controllable and reversible tunability of the intercavity gap separation for resonators co-integrated side by side on the same substrate. We demonstrate the occurrence of super-modes not only for a pair of resonators but also for a linear array of three.

\section{MATERIALS AND METHODS}

Fabrication of polymeric micro-lasers on elastomer substrates We have fabricated on-chip yet flexible polymeric photonic molecules consisting of dye-doped WGM resonators on an elastic polymer substrate. These high- $Q$ resonators have been structured next to each other on the substrate, as shown in Figure 1a-1d. For the substrate, we used commercially available polydimethylsiloxane (PDMS, SYLGARD 184 Silicone Elastomer, Dow Corning GmbH, Wiesbaden, Germany). The weight ratio of the elastomer and curing agent was chosen to be 13:1 rather than the commonly used 10:1 ratio in order to enhance the substrate elasticity. PDMS was then poured into a casting mold on a glass slide, which was previously rinsed thoroughly with acetone and isopropanol to obtain a clean and flat surface for the subsequent processes, as shown in Figure 1a. Next, PDMS was cured at room temperature for at least $48 \mathrm{~h}$. Afterwards, the $\sim 2 \mathrm{~mm}$-thick PDMS layer was pulled off the glass slide and used as a substrate, providing a smooth surface for the micro-resonators. In order to promote adhesion of the resonators, the PDMS surface was functionalized before further structuring steps ${ }^{45,46}$. The substrate was activated using an oxygen plasma treatment (NanoB, Diener electronic $\mathrm{GmbH}$ Co. $\mathrm{KG}$, Ebhausen, Germany) for $2 \mathrm{~min}$ at $30 \mathrm{~W}$ with a pressure of $0.3 \mathrm{mbar}$ and an oxygen flow of $10 \mathrm{sccm}$ to change the
PDMS surface from hydrophobic to hydrophilic ${ }^{47}$. The substrate was then immersed in a $1 \mathrm{mM}$ solution consisting of 3-methacryloxypropyltrimethoxysilane and ethanol for $30 \mathrm{~min}$ and afterwards was rinsed with ethanol and distilled water to prevent the accumulation of residues on the substrate (Figure 1b). For active resonators, the negative-tone resist OrmoComp (Micro Resist Technology $\mathrm{GmbH}$, Berlin, Germany) was doped with $20 \mu \mathrm{mol}$ of the laser dye Pyrromethene 597 (Radiant Dyes Laser \& Accessoires GmbH, Wermelskirchen, Germany) per gram photoresist ${ }^{48}$. The dye-doped resist was then drop casted onto the PDMS substrate and covered with a coverslip, preventing the photoresist from falling off the substrate. For three-dimensional (3D) lithography ${ }^{48}$, a commercial DLW system (Photonic Professional, Nanoscribe GmbH, Eggenstein-Leopoldshafen, Germany) equipped with a frequency-doubled fiber laser was used. The polymerization of OrmoComp by two-photon absorption leads to covalent binding between the silane and the acrylates of the photoresist, and makes it possible to overcome the poor natural photoresist adhesion on PDMS. In this way, micro-cavities anchored on the PDMS surface can be structured using 3D lithography, as illustrated in Figure 1c. After exposure, the unpolymerized photoresist is removed by chemically developing the samples using a solution of $50 \%$ methyl isobutyl ketone and 50\% isopropanol for $12 \mathrm{~min}$. After subsequent rinsing with acetone and isopropanol and drying in a nitrogen atmosphere, the polymerized resonators remain on the PDMS substrate, as shown in Figure 1d.

Using this fabrication procedure, arrays of closely spaced microresonators can be structured in arbitrary arrangements. The achievable inter-cavity gaps between adjacent polymer resonators are typically near $1 \mu \mathrm{m}$. This value is actually larger than the minimum gap size achieved with electron beam lithography of polymeric WGM cavities (on the order of $300 \mathrm{~nm}$ ). However, even this narrow gap size must be further reduced post fabrication to below $200 \mathrm{~nm}$ for the successful optical coupling of resonators ${ }^{20}$. The advantage of DLW, however, is that non-conductive substrates (for example, elastomers) can be used, facilitating tunable coupling.

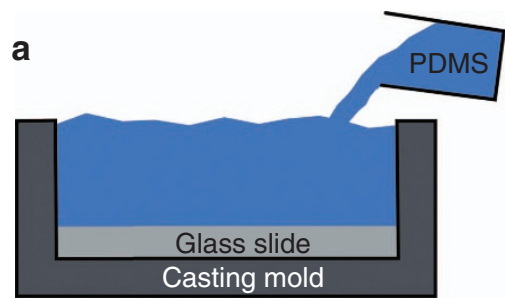

Pouring PDMS onto glass slide

C

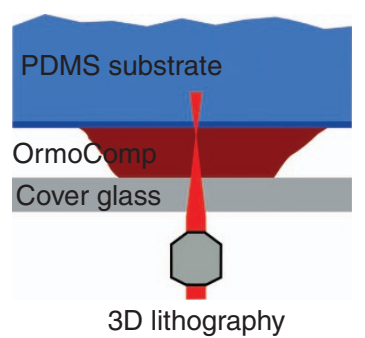

b

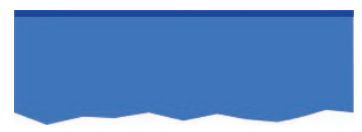

PDMS substrate with flat activated surface

d

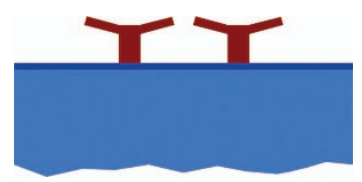

Dye-doped micro-cavities fixed on PDMS

Figure 1 Illustration of the process steps to fabricate polymer WGM micro-lasers on PDMS. A PDMS substrate with a flat surface is fabricated by pouring silicone mixed with curing agent into a mold on a glass slide (a). To enhance adhesion, the PDMS surface is activated using $\mathrm{O}_{2}$ plasma etching and silanization (b). Polymer micro-resonators are structured in sandwich-type geometry by DLW (c). The unpolymerized resist is removed by chemical development, yielding micro-lasers on a flexible substrate (d). 
Mechanical tuning of the coupling gap

To tune the coupling gap, the flexible, non-auxetic PDMS substrate is fixed between two clamps and stretched using a micrometer screw. By orienting the major axis of the PM perpendicular to the stretching direction, the lateral contraction of the elastomer substrate can be exploited in order to decrease the inter-cavity gaps. This coupling mechanism is illustrated in Figure 2a, with scanning electron micrographs of two and three adjacent goblet-shaped micro-cavities shown in Figure $2 \mathrm{~b}$ and 2c.

In order to control the coupling gap, the lateral substrate shrinkage has been calibrated. For this purpose, a grid was drawn onto an identical PDMS substrate, and different stretching forces were applied on the substrate. The lateral substrate shrinkage with respect to the substrate expansion was determined from photographic images. A linear relation between the substrate expansion and the coupling gap was found. More information about the substrate calibration can be found in the Supplementary Information.

Spatially resolved spectroscopy of (super-) modes

Optical characterization of the PM lasers was performed by microphotoluminescence ( $\mu$-PL) spectroscopy. The micro-lasers were pumped with $10 \mathrm{~ns}$ pulses of a frequency-doubled neodymium-doped yttrium orthovanadate $\left(\mathrm{Nd}: \mathrm{YVO}_{4}\right)$ laser at a wavelength of $532 \mathrm{~nm}$ and a repetition rate of $20 \mathrm{~Hz}$. Pulsed excitation with pump pulses shorter than the typical intersystem crossing rate of the dyes, $k_{\mathrm{IC}}^{-1} \sim 100 \mathrm{~ns}^{49}$, is required for investigating lasing in micro-cavities because it can prevent fluorescence quenching of the dyes owing to triplet formation ${ }^{50}$. The pump beam is focused at an incident angle of $\sim 45^{\circ}$ to a spot size of $\sim 100 \mu \mathrm{m}$, which allows the homogenous illumination of all cavities involved in the coupling process. The resonators can simultaneously be imaged on a camera and on the entrance slit of a spectrometer using optics that include a longworking distance microscope objective (magnification of $50 \times$ and numerical aperture of 0.4). In this way, the PL emission can be analyzed in a spectrometer (focal length $=0.5 \mathrm{~m}, 1200$ or 2400 lines $\mathrm{mm}^{-1}$ grating) equipped with a charge-coupled devise camera. Illustration schemes of the setup can be found in earlier publications ${ }^{20,51}$. By orienting the sample so that the major axis of the $\mathrm{PM}$ is aligned parallel to the entrance slit (Figure 3), the $\mu$-PL setup allows the emitted light from the resonators to be not only spectrally resolved but also spatially resolved: WGM lasing emission from the distant rims of the cavities as well as from the coupling region can be simultaneously recorded and can enable the distinction of noncoupled lasing modes of the individual cavities and lasing supermodes of the coupled photonic molecule.

\section{RESULTS AND DISCUSSION}

To investigate the coupling effects among polymer micro-resonators, two different experiments were performed. First, the results of the tunable coupling of two size-mismatched micro-lasers are presented; then, we show the formation of even larger PMs consisting of a linear chain of three equally sized micro-cavities.

\section{Flexible formation of photonic molecules consisting of two} micro-lasers

To demonstrate the flexible formation of PMs, goblet-shaped microresonators with diameters of 30 and $50 \mu \mathrm{m}$ were structured on a PDMS substrate with an inter-cavity gap of $3 \mu \mathrm{m}$. The cavities were pumped with an energy density of $960 \mu \mathrm{J} \mathrm{pulse}^{-1} \mathrm{~cm}^{-2}$. The lasing thresholds for single resonators of these types were measured to be as low as 230 (resonators with $50 \mu \mathrm{m}$ diameter) and $430 \mu \mathrm{J}$ pulse $^{-1} \mathrm{~cm}^{-2}$ (resonators with $30 \mu \mathrm{m}$ diameter). Hence, in our experiment, the
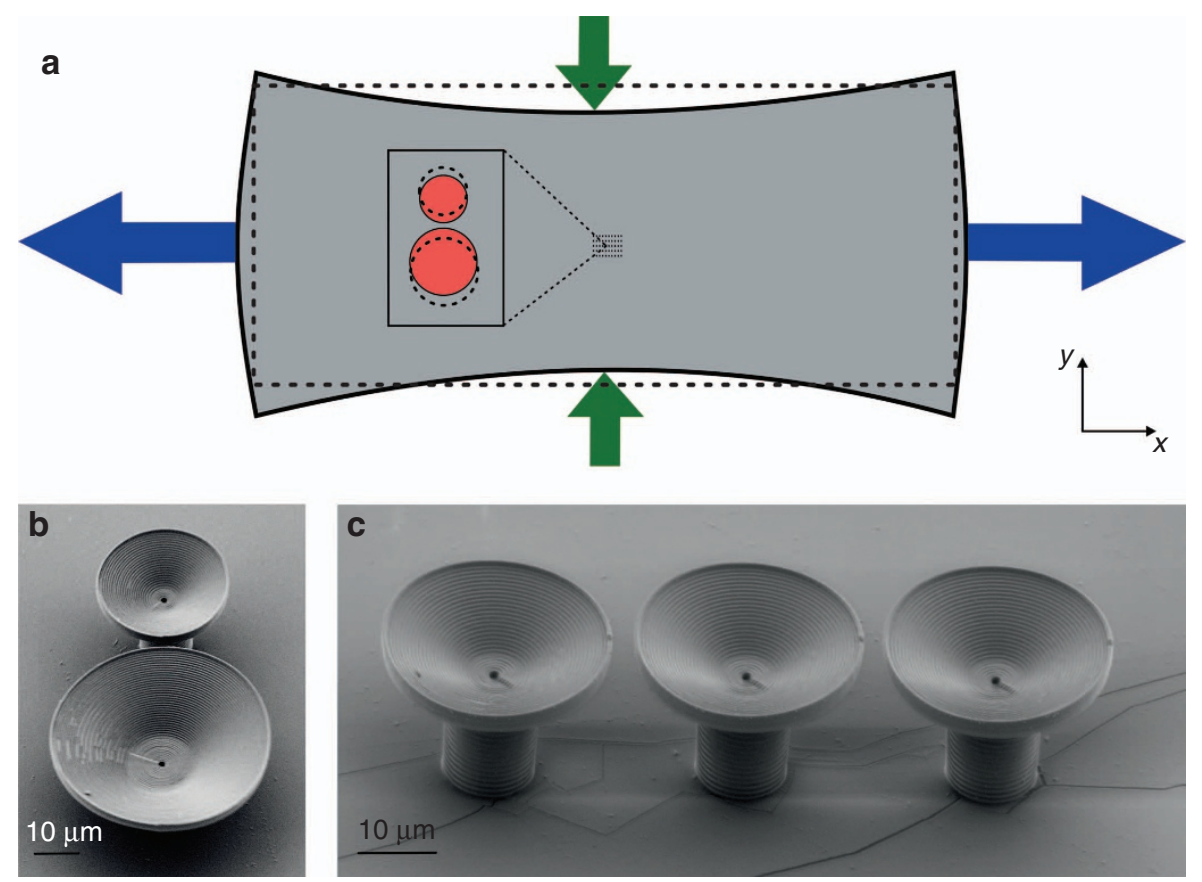

Figure 2 (a) Schematic of the tunable coupling mechanism by stretching a PDMS substrate (blue arrows) and exploiting the lateral shrinkage (green arrows). The dotted lines show the pre-stretching state of the substrate and the inter-cavity distance, whereas the solid line represents the substrate after applying a force in the $x$ direction. The red circles illustrate the resonators with a reduced gap after expansion. (b) Scanning electron micrograph of two goblet-shaped polymeric micro-resonators fabricated by DLW on a PDMS elastomer substrate with diameters of 30 and $50 \mu \mathrm{m}$, and a designed inter-cavity gap of $3 \mu \mathrm{m}$. (c) Scanning electron micrograph of three equal-sized polymeric micro-goblets with diameters of $30 \mu \mathrm{m}$. 


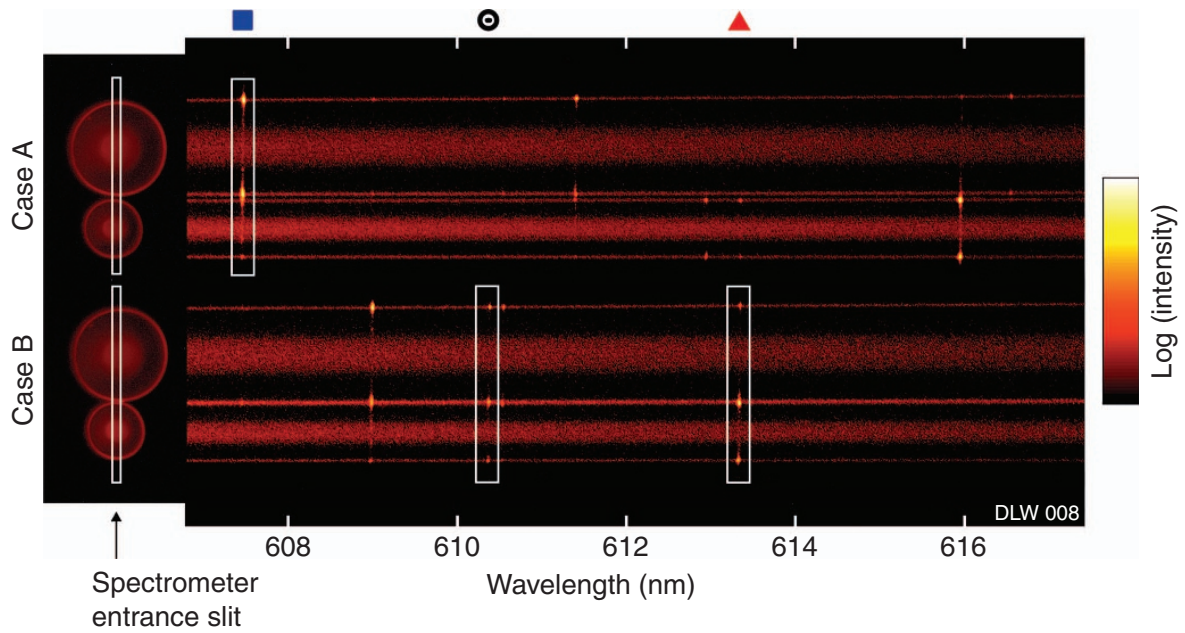

Figure 3 Spatially resolved $\mu$-PL spectra of micro-lasers with diameters of 30 and $50 \mu \mathrm{m}$ indicating the localization of WGM lasing modes for the uncoupled case (Case A) and the coupled case (Case B). Whereas in Case A, the cavities are considered to be independent micro-lasers with modes in either cavity, Case B shows the coupled system with lasing super-modes extending over both resonators.

resonators are pumped well above the typical lasing threshold energy density. In order to determine the quality factors of the cavities, transmission measurements through a tapered glass fiber approaching the resonators were performed, yielding $Q$ factors up to $7.9 \times 10^{4}$ $(50 \mu \mathrm{m}$ diameter $)$ and $2.3 \times 10^{4}(30 \mu \mathrm{m}$ diameter $)$. With this method, we also confirmed the free spectral ranges expected based on theory $\left(\mathrm{FSR}_{50 \mu \mathrm{m}}=1.69 \mathrm{~nm}, \mathrm{FSR}_{30 \mu \mathrm{m}}=2.77 \mathrm{~nm}\right)$. For more details on the characteristics of the resonators, see the Supplementary Information.

Two of the recorded spatially resolved $\mu$-PL spectra are shown in Figure 3. Case A represents the spectrum taken at the designed initial gap distance of $3 \mu \mathrm{m}$. High intensities of the WGM lasing emission can clearly be localized at the rim region of the cavities. The background fluorescence in the middle region originates from the pedestal, which is also dye-doped. The modes are clearly localized either in the upper or in the lower cavity, and both resonators can thus be considered as independent micro-lasers.

Uncoupled lasing modes have similar intensities at the rims of one cavity but no or significantly less intensity in the other cavity. This situation is illustrated for the mode at $607.46 \mathrm{~nm}$, framed with a white rectangle and marked with a blue square in Case A. The small residual intensity at the lower rim of the lower resonator can be explained through scattering of the radiated laser emission from the upper resonator at this rim region. Clearly, the intensities in the upper and lower resonator are substantially different, showing that the mode is solely resonant in the upper cavity. To underline this point, the cavities were also pumped individually before the coupling experiment. In that case also, no lasing mode was observed in the lower resonator at this spectral position, proving that the residual intensity in the lower cavity is not due to a resonant mode but rather to the explained scattering. Consequently, the initial gap is still too large for photon exchange among the cavities.

Reducing the coupling gap by the above method actually leads to a photon exchange among the cavities and the formation of a PM. The mode localization in the coupled micro-laser system changes significantly compared with the uncoupled case (Case A), as lasing modes with equal intensities at both opposing ends as well as at the central region of the PM are observed. These modes, which extend over both resonators, can be considered as delocalized super-modes. An example for the coupled case with a coupling gap of $\sim 65 \mathrm{~nm}$ is illustrated in Case B in Figure 3. The appearance of super-mode lasing for reduced gap widths proves the successful formation of a coupled micro-cavity system.

Concerning the lasing characteristics of the super-modes, we find no significant difference in lasing threshold compared with the uncoupled, single resonators. Referring to the relation between quality factor and lasing threshold stated by Mazumder et $a l^{52}$, we conclude that the quality factor of the super-modes of photonic molecules is on the same order of magnitude as for modes in single resonators $\left(\sim 10^{4}\right)$.

\section{Flexible gap tuning and its influence on the modal structure}

In the following, we intend to focus on the flexible tuning of the intercavity gap and its impact on the modal structure in the PM. The experimental results nicely illustrate the interplay of the modal optical gain and the Vernier effect ${ }^{21,53,54}$. Single active WGM resonators typically show a rich spectrum of lasing modes where, in addition to the fundamental modes, a multitude of higher-order modes compete for the optical gain ${ }^{11,20,51}$. Moving a second WGM resonator into the evanescent field of the lasing modes leads to optical losses unless a resonance of the second cavity is found at the same wavelength. The former scenario leads to an increasing lasing threshold or even quenching of the lasing, while the latter results in the formation of extended super-modes ${ }^{20}$ (Vernier effect). Of course, the coupling strength and overlap of the modal field with the gain region determine the threshold and intensity of the lasing super-modes. Overall, a reduction of lasing modes is observed starting at the threshold and resulting in single-mode lasing for small gaps ${ }^{20}$. However, we show below that new lasing super-modes also emerge that have not been observed above the threshold in uncoupled resonators.

The scenarios discussed above are illustrated in Figure 3: (i) a supermode occurs where in the uncoupled arrangement a lasing mode was observed only in one of the cavities (example marked by red triangle); (ii) a lasing super-mode arises at a wavelength where no lasing modes were previously observed (example marked by a black circle); and (iii) the attenuation of an individual cavity mode leads to its extinction (example marked by a blue square). More details on the dependence of the modal structure on the coupling gap are reflected in Figure 4a and $4 \mathrm{~b}$. Here we plot the integrated intensities (now on a clearer linear 


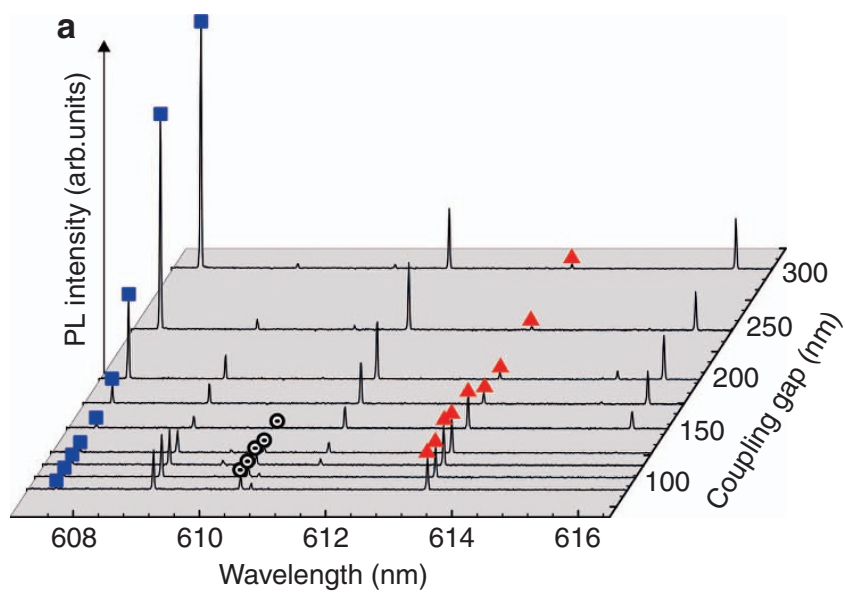

b

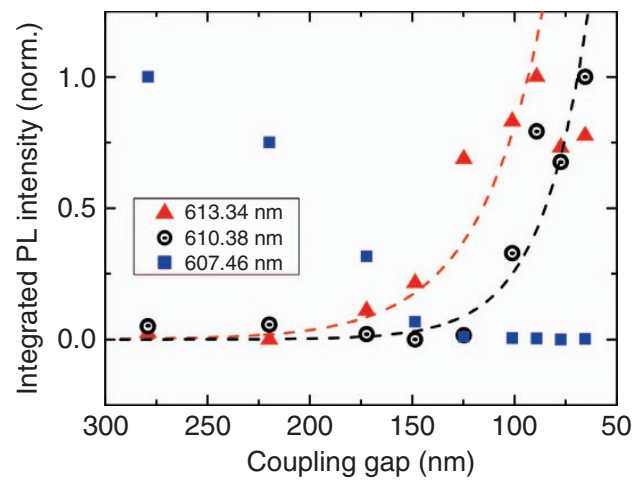

Figure 4 (a) Photoluminescence spectra recorded for different coupling gap widths. The increase (decrease) in the lasing intensities for arising lasing supermodes (vanishing non-resonant lasing modes in the coupled system) can be clearly tracked. (b) For the same example modes marked in Figure 3 (blue square, black circle and red triangle), the integrated lasing-mode intensities are plotted versus the coupling gap. Smooth trends of arising super-modes and vanishing individual modes can be observed for decreasing coupling gaps and attributed to an exponential increase in the coupling strength. To illustrate this point, exponential trend lines are shown for the arising super-modes.

rather than logarithmic scale) of those example modes, illustrating scenarios (i)-(iii) as a function of gap size between the two resonators. To interpret the results, one must consider that a minor part of the observed intensity can be due to light scattering from the neighboring resonator. This fact and how to avoid misinterpretation are discussed above. Furthermore, as the measured intensity is mainly due to laser light scattered from surface imperfections toward the detector (perfect high-Q WGM resonators would not show bright emission), we cannot detect absolute intensity values but rather only trends of their evolution. Nevertheless, the pronounced trends of the lasing intensities in Figure 4 in combination with the above identified localization of the modes (Figure 3) convincingly demonstrate the tunable optical coupling of the two cavities.

Specifically, to record the spectra shown in Figure 4, we used the same cavity pair and experimental conditions used in Figure 3. The spectra are vertically binned over the full extent of the spectrometer slit and plotted for inter-cavity gaps smaller than $300 \mathrm{~nm}$. The change in the photoluminescence intensity is tracked as a function of the coupling gap, which can be tuned with a precision of $12 \mathrm{~nm}$ (Supplementary Information). After each mechanical tuning step, the detection path is realigned for maximum signal.

The mode at $613.34 \mathrm{~nm}$ (red triangles) shows a steep increase with decreasing coupling gap and reflects scenario (i) described above. Here the lasing mode was already visible in one of the cavities before coupling. The mode at $610.38 \mathrm{~nm}$ (black circles) shows a similar rise but has no precursors in the single cavities (scenario (ii)). The super-mode has access to a larger gain volume, boosting it beyond the threshold. The mode located at $607.46 \mathrm{~nm}$ (blue squares) shows a decrease in intensity until complete extinction owing to the increased loss when no matching mode is present in the second cavity (scenario (iii)). By increasing the coupling gap through releasing the substrate strain back to the initial substrate state, the photoluminescence spectrum changes back to the uncoupled case with the same individual lasing modes as before in the individual resonators. This behavior illustrates the reversibility of the applied coupling scheme using elastomer substrates.

The intensity trends documented in Figures 3 and 4 are exponential within experimental uncertainties. To illustrate this point, exponential trend lines (no fits) have been added to the intensity increase of the arising super-modes. The slightly different onset of the two super- modes can be attributed to differences in the overlaps of the electromagnetic fields and therefore different coupling strengths. This behavior is analogous to the reduction of the number of modes with decreasing coupling gap investigated by Grossmann et al ${ }^{20}$. Here the modes with different field overlaps also vanish for different gap widths. The observed exponential increase in intensity can be attributed to the exponential dependence of the inter-cavity photon tunneling rate on the gap size.

In order to understand these experimental findings in more detail, we compared them with theoretical expectations. An analytic expression for the coupling strength between two micro-resonators was derived by Little et $a l^{55}$. They introduced the coupling parameter $\kappa$, the fraction of the amplitude of the electric field coupled from one resonator to the other. Accordingly, $\kappa^{2}$ is also called the 'power coupling coefficient ${ }^{55}$. The coupling parameter $\kappa$ was shown to increase exponentially with a decreasing coupling gap width $g$. On the basis of this theoretical model, we calculated the coupling parameter $\kappa(g)$ for the investigated size-mismatched PM (see Supplementary Information for details). Coupling parameters begin to become sizable for gap separations below $300 \mathrm{~nm}$, with an exponential increase for decreasing gaps. This result is in good accordance with the intensity trends shown in Figure 4. For the vanishing individual modes, the coupling losses reflected by the decreasing lasing intensity increase significantly for gaps smaller than $300 \mathrm{~nm}(\kappa(300 \mathrm{~nm})=0.007)$. For coupling gap sizes near $150 \mathrm{~nm}$ $(\kappa(150 \mathrm{~nm})=0.04)$, losses finally lead to extinction of the mode. In contrast, for matching resonances in both cavities, obviously no losses are introduced. Photon exchange and build-up of the supermodes, however, can only occur for coupling coefficients $\kappa$ well above zero. Thus, for the same magnitude of coupling as above $(\kappa(150 \mathrm{~nm}))$, the super-mode intensity begins to increase in agreement with the experimental observation.

\section{Flexible formation of photonic molecules consisting of three micro-lasers}

Thus far, we have concentrated on the formation of a PM consisting of two micro-lasers and the tunability of its coupling gap. Now, we will demonstrate that the approach using elastomer substrates can be 


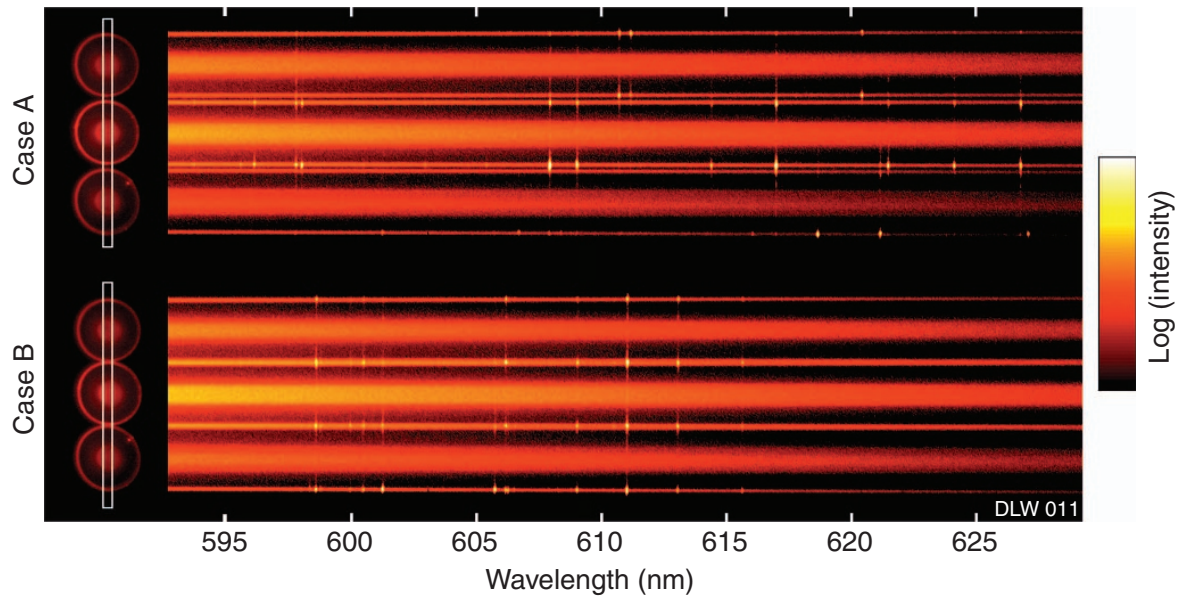

Figure 5 Spatially resolved $\mu$-PL spectra of three equal-sized micro-goblet lasers with diameters of $30 \mu \mathrm{m}$. Individual lasing modes localized in either of the cavities are observed for the initial large coupling gap of $2 \mu \mathrm{m}$ in Case A. As a clear contrast, Case B represents the coupled case for a coupling gap separation reduced to $\sim 55 \mathrm{~nm}$, with lasing super-modes extending over all three micro-lasers.

extended to larger cavity arrays. We give below an example of a flexible, on-chip PM consisting of three micro-cavities.

A linear array of three goblet-shaped WGM micro-lasers with equal diameters of $30 \mu \mathrm{m}$ has been fabricated as described above. The cavities are pumped above their lasing threshold with a pump energy density of $1130 \mu \mathrm{J}$ pulse $\mathrm{cm}^{-2}$ similar to the conditions above. Again, spatially resolved $\mu$-PL measurements allow a clear distinction between the uncoupled (Case A) and the coupled case (Case B), as shown in Figure 5. In Case A, again, only modes localized in the individual cavities can be observed. In Case $\mathrm{B}$, the overall spectrum has changed markedly. Reducing the gap from a designed initial gap distance of $2 \mu \mathrm{m}$ to $\sim 55 \mathrm{~nm}$ allows photon exchange among the single micro-lasers. Thus, delocalized super-modes that extend over all resonators involved can be clearly detected, demonstrating the successful optical coupling of the three cavities. On the other hand, many individual lasing modes present in Case A (see, for example, the low-energy part of spectrum) have vanished in Case B, as expected owing to increased optical coupling losses related to the Vernier effect. After releasing the substrate, the coupled-case spectrum reverts to the initial PL spectrum, with the same individual modes as before straining the substrate.

These data prove that our fabrication scheme enables the flexible formation of PMs consisting of three resonators arranged in a linear chain on a polymer chip. As the coupling mechanism and the fabrication are not limited to three micro-resonators, it should be possible to apply this approach to couple an even larger number of cavities.

\section{CONCLUSIONS}

To summarize, we have presented an approach that allows the postfabrication flexible tuning of the coupling gap width between two or three optical micro-resonators. Goblet-shaped polymer WGM microlasers were structured in a linear arrangement on an elastomer substrate by DLW. Then, the substrate was mechanically stretched in order to exploit a lateral substrate contraction perpendicular to the stretching direction. This method allows fine-tuning of the coupling gap with a precision comparable to a nano-positioning piezo system but with the significant advantage that the cavities are arranged on one polymer chip. Coupling of the resonators to photonic molecules was demonstrated by the observation of super-mode lasing in spatially resolved $\mu$-PL spectroscopy. Super-modes (non-super-modes) show a distinct increase (vanishing) of lasing intensity, which can be attributed to the exponential increase of the coupling strength and the inter-cavity tunneling rate.

The presented coupling approach can in principle be further extended to realize even larger coupled on-chip cavity arrays. In this way, our approach, together with tapered fiber coupling or $3 \mathrm{D}$ polymer waveguide structuring ${ }^{56}$, could enable the fabrication of tunable CROW devices. Of course, the focus of the work presented here is not the technical improvement of existing CROWs. However, we demonstrated that new and important degrees of freedom such as flexibility and tunability can be added. There is the prospect that novel features associated not only with CROWs but also with other photonic structures arise owing to this tunability evoked by the use of elastomer substrates. Both fundamental aspects such modal splitting or slow light propagation and applications such as tunable filters or switches can be addressed in a more elaborate way.

\section{CONFLICT OF INTEREST}

The authors declare no conflict of interest.

\section{ACKNOWLEDGEMENTS}

This work has been financially supported by the Karlsruhe School of Optics and Photonics (KSOP). TS, SK and SFW are pursuing their PhD degrees within KSOP. SK acknowledges financial support from the Carl Zeiss foundation. The fabrication was performed with support of the Nanostructure Service Laboratory, a service-oriented facility within the CFN at KIT. We acknowledge support by Deutsche Forschungsgemeinschaft and the Open Access Publishing Fund of the Karlsruhe Institute of Technology.

1 Bose S, Angelakis DG, Burgarth D. Transfer of a polaritonic qubit through a coupled cavity array. J Mod Opt 2006; 54: 2307-2314.

2 Hartmann MJ, Brandão FGSL, Plenio MB. Strongly interacting polaritons in coupled arrays of cavities. Nat Phys 2006; 2: 849-855.

3 Hartmann MJ, Brandão FGSL, Plenio MB. Quantum many-body phenomena in coupled cavity arrays. Laser Photonics Rev 2008; 2: 527-556.

4 Vollmer F, Arnold S. Whispering-gallery-mode biosensing: label-free detection down to single molecules. Nat Methods 2008; 5: 591-596. 
5 Lu TW, Lee PT. Ultra-high sensitivity optical stress sensor based on double-layered photonic crystal microcavity. Opt Express 2009; 17: 1518-1526.

6 Guzatov DV, Woggon U. Coupled microsphere clusters for detecting molecule's dipole moment orientation. App/ Phys Lett 2009; 94: 241104.

7 Zhang XW, Ren LQ, Wu X, Li H, Liu L et al. Coupled optofluidic ring laser for ultrahighsensitive sensing. Opt Express 2011; 19: 22242-22247.

8 Santiago-Cordoba MA, Boriskina SV, Vollmer F, Demirel MC. Nanoparticle-based protein detection by optical shift of a resonant microcavity. Appl Phys Lett 2011; 99: 073701

9 Ta VD, Chen R, Ma L, Ying YJ, Sun HD. Whispering gallery mode microlasers and refractive index sensing based on single polymer fiber. Laser Photonics Rev 2013; 7: 133-139.

10 Yang L, Carmon T, Min B, Spillane SM, Vahala KJ. Erbium-doped and Raman microlasers on a silicon chip fabricated by the sol-gel process. App/ Phys Lett 2005; 86: 091114.

11 Grossmann T, Schleede S, Hauser M, Christiansen MB, Vannahme C et al. Low-threshold conical microcavity dye lasers. Appl Phys Lett 2010; 97: 063304.

12 Chen R, Ling B, Sun XW, Sun HD. Room temperature excitonic whispering gallery mode lasing from high-quality hexagonal ZnO microdisks. Adv Mater 2011; 23: 2199-2204.

13 Djordjev K, Choi SJ, Choi SJ, Dapkus PD. Microdisk tunable resonant filters and switches. IEEE Photonics Technol Lett 2002; 14: 828-830.

14 Rabiei P, Steier WH, Zhang C, Dalton LR. Polymer micro-ring filters and modulators. J Light Technol 2002; 20: 1968-1975.

15 Savchenkov AA, Ilchenko VS, Matsko AB, Maleki L. High-order tunable filters based on a chain of coupled crystalline whispering gallery-mode resonators. IEEE Photonics Technol Lett 2005; 17: 136-138.

16 Yang SC, Wang Y, Sun HD. Advances and prospects for whispering gallery mode microcavities. Adv Opt Mater 2015; 3: 1136-1162.

17 Vahala KJ. Optical microcavities. Nature 2003; 424: 839-846.

18 Rakovich YP, Donegan JF. Photonic atoms and molecules. Laser Photonics Rev 2010; 4: 179-191.

19 Bayer M, Gutbrod T, Reithmaier JP, Forchel A, Reinecke TL et al. Optical modes in photonic molecules. Phys Rev Lett 1998; 81: 2582-2585.

20 Grossmann T, Wienhold T, Bog U, Beck T, Friedmann C et al. Polymeric photonic molecule super-mode lasers on silicon. Light Sci Appl 2013; 2 e82, doi:10.1038/lsa.2013.38.

21 Lee W, Li H, Suter JD, Reddy K, Sun YZ et al. Tunable single mode lasing from an on-chip optofluidic ring resonator laser. Appl Phys Lett 2011; 98: 061103.

22 Ta VD, Chen R, Sun HD. Coupled polymer microfiber lasers for single mode operation and enhanced refractive index sensing. Adv Opt Mater 2014; 2: 220-225.

23 Dai DX. Highly sensitive digital optical sensor based on cascaded high- $Q$ ring-resonators. Opt Express 2009; 17: 23817-23822.

24 Jin L, Li MY, He JJ. Highly-sensitive silicon-on-insulator sensor based on two cascaded micro-ring resonators with Vernier effect. Opt Commun 2011; 284: 156-159.

$25 \mathrm{Wu}$ X, Li H, Liu LY, Xu L. Unidirectional single-frequency lasing from a ring-spiral coupled microcavity laser. Appl Phys Lett 2008; 93: 081105.

26 Chern GD, Tureci HE, Stone AD, Chang RK, Kneissl M et al. Unidirectional lasing from InGaN multiple-quantum-well spiral-shaped micropillars. App/ Phys Lett 2003; 83: 1710-1712.

27 Boriskina SV. Theoretical prediction of a dramatic $Q$-factor enhancement and degeneracy removal of whispering gallery modes in symmetrical photonic molecules. Opt Lett 2006; 31: 338-340.

28 Benyoucef M, Shim JB, Wiersig J, Schmidt OG. Quality-factor enhancement of supermodes in coupled microdisks. Opt Lett 2011; 36: 1317-1319.

29 Hryniewicz JV, Absil PP, Little BE, Wilson RA, Ho PT. Higher order filter response in coupled microring resonators. IEEE Photonics Technol Lett 2000; 12: 320-322.

30 Xia FN, Rooks M, Sekaric L, Vlasov Y. Ultra-compact high order ring resonator filters using submicron silicon photonic wires for on-chip optical interconnects. Opt Express 2007; 15: 11934-11941.

31 Chremmos I, Uzunoglu N. Reflective properties of double-ring resonator system coupled to a waveguide. IEEE Photonics Technol Lett 2005; 17: 2110-2112.

32 Boriskina SV. Spectrally engineered photonic molecules as optical sensors with enhanced sensitivity: a proposal and numerical analysis. J Opt Soc Am B 2006; 23: 1565-1573.

33 Poon JKS, Zhu L, DeRose GA, Yariv A. Transmission and group delay of microring coupled-resonator optical waveguides. Opt Lett 2006; 31: 456-458.

34 Xia FN, Sekaric L, Vlasov Y. Ultracompact optical buffers on a silicon chip. Nat Photonics 2007; 1: 65-71.
35 Melloni A, Morichetti F, Martinelli M. Linear and nonlinear pulse propagation in coupled resonator slow-wave optical structures. Opt Quantum Electron 2003; 35: 365-379.

36 Poon JKS, Scheuer J, Xu Y, Yariv A. Designing coupled-resonator optical waveguide delay lines. J Opt Soc Am B 2004; 21: 1665-1673.

37 Tian K, Arora W, Takahashi S, Hong J, Barbastathis G. Dynamic group velocity control in a mechanically tunable photonic-crystal coupled-resonator optical waveguide. Phys Rev B 2009; 80: 134305 .

38 Lee MCM, Wu MC. MEMS-actuated microdisk resonators with variable power coupling ratios. IEEE Photonics Technol Lett 2005; 17: 1034-1036.

39 Chollet F. Devices based on co-integrated MEMS actuators and optical waveguide: a review. Micromachines 2016; 7: 18.

40 Park W, Lee JB. Mechanically tunable photonic crystal structure. Appl Phys Lett 2004; 85: 4845-4847.

$41 \mathrm{Du}$ H, Chau FS, Zhou GY. Mechanically-tunable photonic devices with on-chip integrated MEMS/NEMS actuators. Micromachines 2016; 7: 69.

42 Popović MA, Barwicz T, Watts MR, Rakich PT, Socci L et al. Multistage high-order microring-resonator add-drop filters. Opt Lett 2006; 31: 2571-2573.

43 Beck T, Schloer S, Grossmann T, Mappes T, Kalt H. Flexible coupling of high- $Q$ goblet resonators for formation of tunable photonic molecules. Opt Express 2012; 20: 22012-22017.

44 Peng B, Özdemir ŞK, Zhu JG, Yang L. Photonic molecules formed by coupled hybrid resonators. Opt Lett 2012; 37: 3435-3437.

45 Baldacchini T, LaFratta CN, Farrer RA, Teich MC, Saleh BEA et al. Acrylic-based resin with favorable properties for three-dimensional two-photon polymerization. J Appl Phys 2004; 95: 6072-6076.

46 Buestrich R, Kahlenberg F, Popall M, Dannberg P, Müller-Fiedler R et al. ORMOCERs for optical interconnection technology. J Sol-Gel Sci Technol 2001; 20: 181-186.

47 Chen WQ, Lam RHW, Fu JP. Photolithographic surface micromachining of polydimethylsiloxane (PDMS). Lab Chip 2012; 12: 391-395.

48 Grossmann T, Schleede S, Hauser M, Beck T, Thiel M et al. Direct laser writing for active and passive high- $Q$ polymer microdisks on silicon. Opt Express 2011; 19: 11451-11456.

49 Grivas C, Pollnau M. Organic solid-state integrated amplifiers and lasers. Laser Photonics Rev 2012; 6: 419-462.

50 Kurian A, George NA, Paul B, Nampoori VPN. Vallabhan CPG. Studies on fluorescence efficiency and photodegradation of rhodamine 6G doped PMMA using a dual beam thermal lens technique. Laser Chem 2002; 20: 99-110.

51 Flatae AM, Burresi M, Zeng H, Nocentini S, Wiegele S et al. Optically controlled elastic microcavities. Light Sci App/ 2015; 4: e282, doi:10.1038/lsa.2015.55.

52 Mazumder MM, Chen G, Chang RK, Gillespie JB. Wavelength shifts of dye lasing in microdroplets: effect of absorption change. Opt Lett 1995; 20: 878-880.

53 Shang L, Liu LY, Xu L. Single-frequency coupled asymmetric microcavity laser. Opt Lett 2008; 33: 1150-1152.

54 Boeck R, Jaeger NAF, Rouger N, Chrostowski L. Series-coupled silicon racetrack resonators and the Vernier effect: theory and measurement. Opt Express 2010; 18: $25151-25157$.

55 Little BE, Chu ST, Haus HA, Foresi J, Laine JP. Microring resonator channel dropping filters. J Light Technol 1997; 15: 998-1005.

56 Schumann M, Bückmann T, Gruhler N, Wegener M, Pernice W. Hybrid 2D-3D optical devices for integrated optics by direct laser writing. Light Sci App/ 2014; 3 e175, doi:10.1038/Isa.2014.56.

\begin{abstract}
(c) (i) $(-)$ This work is licensed under a Creative Commons AttributionBY NC ND NonCommercial-NoDerivs 4.0 International License. The images or other third party material in this article are included in the article's Creative Commons license, unless indicated otherwise in the credit line; if the material is not included under the Creative Commons license, users will need to obtain permission from the license holder to reproduce the material. To view a copy of this license, visit http:// creativecommons.org/licenses/by-nc-nd/4.0/
\end{abstract}

(C) The Author(s) 2017

Supplementary Information for this article can be found on the Light: Science \& Applications' website (http://www.nature.com/lsa). 